\title{
Asymmetric Conjugate Addition Reactions of Meldrum's Acid Derived Acceptors Employing Chiral Phosphoramidite Ligands
}

\section{Takumi Watanabe, Thomas F. Knöpfel and Erick M. Carreira*}

Laboratorium für Organische Chemie

ETH-Hönggerberg HCI H335

CH-8093 Zürich, Switzerland.

Carreira@org.chem.ethz.ch

Experimental

General Information:

For the reactions, solvents were purified by distillation and dried by passage over activated alumina under an argon atomosphere $\left(\mathrm{H}_{2} \mathrm{O}\right.$ content $<30 \mathrm{ppm}$, Karl-Fischer titration). For flash chromatography and extractions technical grade solvents were used, which were distilled prior to use. All chemicals were purchased from Aldrich or Fluka and used as received.

Chromatographic purification was performed as flash chromatography using Merck silica gel 60 with 0.4 bar pressure.

TLC was performed on Merck silica gel 60 F254 TLC glass plates and visualized with UV light and/or permanganate stain.

${ }^{1}$ H-NMR spectra were recorded on a VARIAN Mercury $300 \mathrm{MHz}$ spectrometer in chloroform-d. All signals are reported in ppm relative to TMS with the internal solvent signal $\left(\mathrm{CDCl}_{3}: 7.26\right)$ as standard. The data is reported as ( $\mathrm{s}=$ singlet, $\mathrm{d}=$ doublet, $\mathrm{t}=$ triplet, $\mathrm{m}=$ =multiplet or unsolved signal, coupling constant(s) in $\mathrm{Hz}$, integration).

${ }^{13} \mathrm{C}$-NMR spectra were recorded with ${ }^{1} \mathrm{H}$-decoupling on VARIAN Mercury $75 \mathrm{MHz}$ spectrometer in chloroform-d, all signals are reported in ppm relative to TMS with the internal solvent signal $\left(\mathrm{CDCl}_{3}\right.$ : 77.0) as standard.

Infrared spectra were recorded on a Perkin-Elmer spectrum RX-I FT-IR spectrometer as thin films or $\mathrm{KBr}$ pellets. The data is being reported as absorption maxima $\left(v, \mathrm{~cm}^{-1}\right)$ with corresponding 


\section{Supplementary Material}

characteristic intensity ( $\mathrm{w}=\mathrm{weak}, \mathrm{m}=$ medium, $\mathrm{s}=$ strong).

Melting points were measured on a Buechi 510 melting point apparatus using open glass capillaries and are uncorrected.

Mass spectrometric measurements were performed by the mass spectrometry service of the LOC at the ETHZ.

Combustion analyses were performed by the Mikroelementarische Laboratorium of the LOC at the ETHZ.

Enantiomeric excesses were determined by chiral HPLC analysis of derivatives of adducts (anilides of corresponding monocarboxylic acid) with Merck-Hitachi D-7000 system. Solvent mixtures, conditions, retention times and Chiracel columns used are given in parentheses.

Optical rotation $[\alpha]^{25}$ were measured by Jasco DID-1000 or DID-1030 Polarimeter, $10 \mathrm{~cm} 1 \mathrm{~mL}$ cell. Concentration (c, g/100 mL), solvent and ee of the each sample are given in parentheses. 


\section{Supplementary Material}

Preparation of chiral ligand

$(R, R)$-Bis-(1-cyclohexyl-ethyl)-(3,5-dioxa-4-phospha-cyclohepta[2,1-a;3,4-a']-(S)-dinaphthalen4-yl)-amine (3).

A chiral ligand $\mathbf{3}$ was synthesized according to a reported procedure ${ }^{1)}$. The title compound (128.6 $\mathrm{mg}$, $0.234 \mathrm{mmol})$ was obtained as a white foam in $68 \%$ yield from $98.5 \mathrm{mg}(0.344 \mathrm{mmol})$ of BINOL and $47.2 \mathrm{mg}(0.344 \mathrm{mmol})$ of $(R, R)$-Bis-(1-cyclohexyl-ethyl)-amine $\left.{ }^{2}\right)[\alpha]^{25}{ }_{\mathrm{D}}+301^{\circ}\left(\mathrm{c}=0.93, \mathrm{CHCl}_{3}\right)^{1} \mathrm{H}$ NMR $\delta$ 0.60-2.10 (m, 28H), $2.96(\mathrm{~m}, 2 \mathrm{H}), 7.17-7.28(\mathrm{~m}, 3 \mathrm{H}), 7.35-7.50(\mathrm{~m}, 5 \mathrm{H}), 7.84-7.98(\mathrm{~m}, 4 \mathrm{H})$;

${ }^{13} \mathrm{C}$ NMR $\delta$ 18.9, 26.4, 26.5, 26.6, 26.8, 27.0, 29.67, 29.72, 29.9, 31.6, 32.2, 43.1, 54.7, 54.8, 121.3, $121.6,122.4,123.99,124.05,124.4,125.5,125.7,127.0,128.0,128.1,129.0,129.1,130.0,130.2$, 131.2, 132.6, 149.8, 150.4, 150.6; FTIR (thin film): 2924 (s), 1733 (w), 1621 (w), 1592 (m), 1506 (m), 1463 (s), 1448 (m), 1373 (m), 1329 (s), 1230 (s), 1072 (m), 941 (s), 821 (m); HRMS (FT MALDI) calcd for $\mathrm{C}_{17} \mathrm{H}_{24} \mathrm{NO}_{4}(\mathrm{MH})^{+} 552.3031$ found 552.3023 .

Preparation of Meldrum's alkylidenes.

All the substrates except for $\mathbf{1} \mathbf{i}$ have been reported in literature $\left(\mathbf{1 a}, \mathbf{c}, \mathbf{d}, \mathbf{f}, \mathbf{k}^{3)}, \mathbf{1 b}, \mathbf{l}^{4)}, \mathbf{1} \mathbf{e}, \mathbf{h}^{5)}, \mathbf{1 g}^{(6)}, \mathbf{1}^{\mathbf{7}}\right)$. The compound $1 \mathbf{i}$ was prepared according to the method reported by Bigi et al. ${ }^{3)}$.

\section{2,2-Dimethyl-5-(4-trifluoromethyl-benzylidene)-[1,3]dioxane-4,6-dione (1i)}

White powder: $\mathrm{mp} 134-135^{\circ} \mathrm{C} ;{ }^{1} \mathrm{H}$ NMR $\delta 1.83(\mathrm{~s}, 6 \mathrm{H}), 7.72(\mathrm{~d}, J=7.2 \mathrm{~Hz}, 2 \mathrm{H}), 8.06(\mathrm{~d}, J=7.2 \mathrm{~Hz}, 2 \mathrm{H})$, 8.44 (s, 1H); ${ }^{13} \mathrm{C}$ NMR $\delta 27.8,104.9,117.2,123.3,125.4,132.7,133.9,134.7,155.6,159.0,162.3$; FTIR (KBr): 1733 (s), 1631 (s), 1386 (m), 1327 (m), 1296 (m), 1202 (m), 1171 (m), 1124 (m), 1066 (m), 1037 (w), 933 (w), $842(\mathrm{~m})$; MS (ESI neg.) calcd for $\mathrm{C}_{14} \mathrm{H}_{11} \mathrm{~F}_{3} \mathrm{O}_{4}(\mathrm{M}) 300.1$ found 300.0; Anal. Calcd for $\mathrm{C}_{14} \mathrm{H}_{11} \mathrm{~F}_{3} \mathrm{O}_{4}$ : C, 56.01; H, 3.69. Found: $\mathrm{C}, 55.72 ; \mathrm{H}, 3.50$.

General procedure for the conjugate addition of diethylzinc to Meldrum's alkylidenes

To a solution of $3(5.5 \mathrm{mg}$; $9.97 \mu \mathrm{mol}, 6 \mathrm{~mol} \%)$ and $\mathrm{Cu}\left(\mathrm{O}_{2} \mathrm{CCF}_{3}\right)_{2}(962.3 \mu \mathrm{g}, 3.32 \mu \mathrm{mol}, 2 \mathrm{~mol} \%)$ in THF $(0.2 \mathrm{~mL})$ was added a 1.2 eq. of $1 \mathrm{M}$ solution of $\mathrm{Et}_{2} \mathrm{Zn}$ in hexane $(199.4 \mu \mathrm{L}, 0.199 \mathrm{mmol})$ and a solution of a Meldrum's alkylidene $(0.166 \mathrm{mmol})$ in $0.3 \mathrm{~mL}$ of THF (or the least amount of THF to dissolve the substrate) at $-78{ }^{\circ} \mathrm{C}$ successively. The reaction was stirred for $3 \mathrm{~h}$ at the temperature, quenched with $0.5 \mathrm{~N} \mathrm{HCl}$. The mixture was extracted with $\mathrm{CH}_{2} \mathrm{Cl}_{2}$ three times. The combined organic layers were washed with brine, dried over $\mathrm{Na}_{2} \mathrm{SO}_{4}$ and concentrated in vacuo. The resulting residue was purified by flash column chromatography $\left(\mathrm{SiO}_{2}\right.$, hexanes:EtOAc) to give the pure product. For some substrates, $1 \mathrm{~mol} \%$ of $\mathrm{Cu}\left(\mathrm{O}_{2} \mathrm{CCF}_{3}\right)_{2}$ and $3 \mathrm{~mol} \%$ of ligand were used. 


\section{Supplementary Material}

A small amount of adduct was converted to the anilide of the corresponding monocarboxylic acid (stirred at $100{ }^{\circ} \mathrm{C}$ for $1 \mathrm{~h}$ in DMF:aniline $=10: 1$ ), of which the ee was determined by HPLC.

\section{(R)-(-)-5-(1-Ethyl-2-methyl-propyl)-2,2-dimethyl-[1,3]dioxane-4,6-dione (2a).}

The compound ( $32.9 \mathrm{mg}, 0.144 \mathrm{mmol}$ ) was obtained from the $1 \mathrm{a}(32.9 \mathrm{mg}, 0.166 \mathrm{mmol})$ in $87 \%$ yield. Physicochemical properties were identical to reported ones $\left.{ }^{8}\right):[\alpha]^{25}-3.98^{\circ}\left(\mathrm{c}=1.065, \mathrm{CHCl}_{3}, 91 \%\right.$ ee $)$. The enantioselectivity was $92 \%$ ee (Daicel Chiracel OD-H column $(15 \mathrm{~cm})$ with hexane:2-propanol=92:8, flow $1 \mathrm{~mL} / \mathrm{min}$, retention time: $8.5 \mathrm{~min}$ : $R$-enantiomer, $9.7 \mathrm{~min}$ : $S$-enantiomer).

The absolute configuration was determined by optical rotation of 3-ethyl-4-pentanol ${ }^{9)}$ derived from $\mathbf{2 a}$ ((i) wet DMF, $100{ }^{\circ} \mathrm{C}$, (ii) $\mathrm{LiAlH}_{4}$ ).

\section{(R)- (-)-5-(1-Cyclohexyl-propyl)-2,2-dimethyl-[1,3]dioxane-4,6-dione (2b).}

The compound ( $37.5 \mathrm{mg}, 0.140 \mathrm{mmol}$ ) was obtained from the $1 \mathrm{~b}(39.6 \mathrm{mg}, 0.166 \mathrm{mmol}$ ) in $84 \%$ yield as a white solid: $\mathrm{mp} 98-99^{\circ} \mathrm{C}$; $[\alpha]^{25} \mathrm{D}-11.9^{\circ}\left(\mathrm{c}=0.125, \mathrm{CHCl}_{3}\right) ;{ }^{1} \mathrm{H}$ NMR $\delta 0.92(\mathrm{t}, J=7.5 \mathrm{~Hz}, 3 \mathrm{H})$, 0.90-1.30 (m, 6H), 1.50-1.80 (m, 6H), 1.93 (m, 1H), 2.25 (ddt, $J=4.5,2.4,9.0 \mathrm{~Hz}, 1 \mathrm{H}), 3.53$ (d, $J=2.4$ $\mathrm{Hz}, 1 \mathrm{H}) ;{ }^{13} \mathrm{C}$ NMR $\delta 13.2,23.3,26.38,26.45,26.6,27.6,28.2,31.6,31.8,39.8,45.8,46.7,104.5$, 165.9; FTIR (KBr): 2930 (s), 1783 (s), 1742 (s), 1386 (m), 1315 (s), 1208 (m), 1057 (w), 993 (m), 907 (w), 874 (m); MS (ESI neg.) calcd for $\mathrm{C}_{15} \mathrm{H}_{23} \mathrm{O}_{4}(\mathrm{M}-\mathrm{H})^{-} 267.2$ found 267.1; Anal. Calcd for $\mathrm{C}_{15} \mathrm{H}_{24} \mathrm{O}_{4}$ : C, 67.14; H, 9.01. Found: C, 67.06; H, 9.05.

The enantioselectivity was $94 \%$ ee (Daicel Chiracel OD-H $(15 \mathrm{~cm})$ column with hexane:2-propanol=92:8, flow $1 \mathrm{~mL} / \mathrm{min}$, retention time: $8.5 \mathrm{~min}: R$-enantiomer, $10.3 \mathrm{~min}$ : $S$-enantiomer).

The absolute configuration was determined by the sign of optical rotation of 3-cyclohexylpentanic acid $^{10)}$ derived from $\mathbf{2 b}$ (wet DMF, $100^{\circ} \mathrm{C}$ ).

\section{(R)-(+)-2,2-Dimethyl-5-(1-phenyl-propyl)-[1,3]dioxane-4,6-dione (2c).}

The compound (41.1 mg, $0.157 \mathrm{mmol})$ was obtained from the 1c (38.6 mg, $0.166 \mathrm{mmol})$ in $94 \%$ yield. Physicochemical properties were as reported ${ }^{8)}:[\alpha]^{25}{ }_{\mathrm{D}}+10.0\left(\mathrm{c}=4.395, \mathrm{CHCl}_{3}, 31 \%\right.$ ee $)$.

and The enantioselectivity was $40 \%$ ee (Daicel Chiracel AD-H column $(25 \mathrm{~cm})$ with hexane:2-propanol=90:10, flow $0.8 \mathrm{~mL} / \mathrm{min}$, retention time: $13.1 \mathrm{~min}$ : $S$-enantiomer, $16.4 \mathrm{~min}$ : $R$-enantiomer)

Absolute configuration was confirmed as follows; the adduct $\mathbf{2 c}$ was converted to 1,3-diphenyl-1-pentanone in 3 steps ((i) wet $\mathrm{DMF}$ for $1 \mathrm{hr}$ at $100{ }^{\circ} \mathrm{C}$ (ii) $\mathrm{SOCl}_{2}$, then N,O-dimethylhydroxylamine hydrochloride and pyridine (iii) $\mathrm{PhLi} / \mathrm{THF}$ ). A condition of chiral columun HPLC to determine the ee of the resulting ketone has been reported by Fu et al. ${ }^{11)}$ 


\section{Supplementary Material}

\section{(R)-(+)-5-[1-(4-Methoxy-phenyl)-propyl]-2,2-dimethyl-[1,3]dioxane-4,6-dione (2d).}

The compound (33.4 $\mathrm{mg}, 0.114 \mathrm{mmol})$ was obtained from $44.4 \mathrm{mg}(0.161 \mathrm{mmol})$ of the substrate $1 \mathrm{~d}$ with $5.30 \mathrm{mg}(9.61 \mu \mathrm{mol})$ and $927 \mu \mathrm{g}(3.20 \mu \mathrm{mol})$ of $\mathrm{Cu}\left(\mathrm{O}_{2} \mathrm{CCF}_{3}\right)_{2}$ in $71 \%$ yield as a colorless oil: $[\alpha]^{25}+4.24\left(\mathrm{c}=1.37\right.$, EtOH, 75\% ee). ${ }^{1} \mathrm{H}$ NMR $\delta 0.93(\mathrm{t}, J=7.4 \mathrm{~Hz}, 3 \mathrm{H}), 1.17(\mathrm{~s}, 3 \mathrm{H}), 1.63(\mathrm{~s}, 3 \mathrm{H})$, $2.06(\mathrm{~m}, 1 \mathrm{H}), 2.25(\mathrm{~m}, 1 \mathrm{H}), 3.61(\mathrm{~m}, 1 \mathrm{H}), 3.67(\mathrm{~d}, J=3.3 \mathrm{~Hz}, 1 \mathrm{H}), 3.77$ (s, 3H), $6.83(\mathrm{~d}, J=8.7 \mathrm{~Hz}, 2 \mathrm{H})$, 7.20 (d, $J=8.7 \mathrm{~Hz}, 2 \mathrm{H}) ;{ }^{13} \mathrm{C}$ NMR $\delta 12.7,26.0,28.2,28.5,47.5,51.1,55.2,105.4,113.9,130.0,131.3$, 158.8, 164.7, 165.9; FTIR (thin film): 2968 (s), 1747 (s), 1612 (m), 1514 (s), 1463 (m), 1393 (m), 1383 (m), 1295 (s), 1251 (s), 1208 (m), 1181 (m), 1034 (m), 998 (m), 890 (w); MS (ESI neg.) calcd for $\mathrm{C}_{16} \mathrm{H}_{19} \mathrm{O}_{5}(\mathrm{M}-\mathrm{H})^{-} 291.1$ found 291.0; Anal. Calcd for $\mathrm{C}_{16} \mathrm{H}_{20} \mathrm{O}_{5}: \mathrm{C}, 65.74 ; \mathrm{H}, 6.90$. Found: $\mathrm{C}, 65.97 ; \mathrm{H}$, 6.97.

The enantioselectivity was $87 \%$ ee (Daicel Chiracel AD-H column $(25 \mathrm{~cm})$ with hexane:2-propanol=90:10, flow $0.8 \mathrm{~mL} / \mathrm{min}$, retention time: $18.8 \mathrm{~min}: S$-enantiomer, $23.8 \mathrm{~min}$ : $R$-enantiomer).

Absolute configuration was confirmed as follows; the adduct was converted to 3-(4-Methoxy-phenyl)-1-phenyl-pentan-1-one in 3 steps ((i) wet DMF for $1 \mathrm{hr}$ at $100{ }^{\circ} \mathrm{C}$ (ii) $\mathrm{SOCl}_{2}$, then N,O-dimethylhydroxylamine hydrochloride and pyridine (iii) $\mathrm{PhLi} / \mathrm{THF}$ ). A condition of chiral columun HPLC to determine the ee of the resulting ketone is the one reported by Fu et al. ${ }^{11)}$

\section{(-)-5-[1-(4-Dimethylamino-phenyl)-propyl]-2,2-dimethyl-[1,3]dioxane-4,6-dione (2e).}

The substrate 1e $(79.2 \mathrm{mg}, 0.332 \mathrm{mmol})$, chiral ligand $(5.50 \mathrm{mg}, 9.97 \mu \mathrm{mol}, 3 \mathrm{~mol} \%)$ and $\mathrm{Cu}\left(\mathrm{O}_{2} \mathrm{CCF}_{3}\right)_{2}(962 \mu \mathrm{g}, 3.32 \mu \mathrm{mol}, 1 \mathrm{~mol} \%)$ were employed and the reaction was quenched by saturated $\mathrm{NH}_{4} \mathrm{Cl}$ aqueous solution. The aqueous layer was extracted with $\mathrm{CH}_{2} \mathrm{Cl}_{2}$ three times. The combined organic layers were dried over $\mathrm{Na}_{2} \mathrm{SO}_{4}$ and concentrated in vacuo. The residue was purified by flash column chromatography $\left(\mathrm{SiO}_{2}\right.$, hexanes:EtOAc=5:1) to give the pure product $(91.5 \mathrm{mg}, 0.300$ mmol $)$ in $90 \%$ yield as a white solid: $\mathrm{mp} 96-97^{\circ} \mathrm{C}$; $[\alpha]^{25}{ }_{\mathrm{D}}-3.96^{\circ}\left(\mathrm{c}=1.13, \mathrm{CHCl}_{3}, 88 \%\right.$ ee $){ }^{1}{ }^{\mathrm{H}} \mathrm{NMR}$ $\delta 0.93(\mathrm{t}, J=7.4 \mathrm{~Hz}, 3 \mathrm{H}), 1.12(\mathrm{~s}, 3 \mathrm{H}), 1.61(\mathrm{~s}, 3 \mathrm{H}), 2.05(\mathrm{~m}, 1 \mathrm{H}), 2.21(\mathrm{~m}, 1 \mathrm{H}), 2.90(\mathrm{~s}, 6 \mathrm{H}), 3.54(\mathrm{~m}$, $1 \mathrm{H}), 3.66(\mathrm{~d}, J=3.0 \mathrm{~Hz}, 1 \mathrm{H}), 6.65(\mathrm{~d}, J=9.0 \mathrm{~Hz}, 2 \mathrm{H}), 7.13(\mathrm{~d}, J=9.0 \mathrm{~Hz}, 2 \mathrm{H}) ;{ }^{13} \mathrm{C}$ NMR $\delta 12.8,26.1$, 28.2, 28.6, 40.6, 47.7, 51.2, 105.4, 112.5, 126.9, 129.5, 149.9, 164.8, 166.2; FTIR (KBr): 2968 (s), 1760 (s), 1738 (s), 1610 (m), 1520 (s), 1457 (m), 1380 (m), 1341 (m), 1293 (s), 1205 (m), 1129 (w), 1003 (m), 820 (m); HRMS (FT MALDI) calcd for $\mathrm{C}_{17} \mathrm{H}_{24} \mathrm{NO}_{4}(\mathrm{MH})^{+} 306.1705$ found 306.1695.

The enantioselectivity was $88 \%$ ee (Daicel Chiracel AD-H column $(25 \mathrm{~cm})$ with hexane:2-propanol=90:10, flow $0.8 \mathrm{~mL} / \mathrm{min}$, retention time: $16.1 \mathrm{~min}$ : minor, $22.1 \mathrm{~min}$ : major)

(+)-5-[1-(4-Chloro-phenyl)-propyl]-2,2-dimethyl-[1,3]dioxane-4,6-dione (2f).

The compound ( $34.0 \mathrm{mg}, 0.115 \mathrm{mmol})$ was obtained from $45.2 \mathrm{mg}(0.172 \mathrm{mmol})$ of the substrate $\mathbf{1 f}$ 


\section{Supplementary Material}

with $5.70 \mathrm{mg}(10.3 \mu \mathrm{mol})$ of ligand 3 and $997 \mu \mathrm{g}(3.44 \mu \mathrm{mol})$ of $\mathrm{Cu}\left(\mathrm{O}_{2} \mathrm{CCF}_{3}\right)_{2}$ in $67 \%$ yield as a colorless oil: $[\alpha]^{25}{ }_{\mathrm{D}}+9.63{ }^{\circ}\left(\mathrm{c}=1.265, \mathrm{CHCl}_{3}, 33 \% \mathrm{ee}\right) ;{ }^{1} \mathrm{H}$ NMR $\delta 0.93(\mathrm{t}, J=7.2 \mathrm{~Hz}, 3 \mathrm{H}), 1.37(\mathrm{~s}, 3 \mathrm{H})$, $1.67(\mathrm{~s}, 3 \mathrm{H}), 2.05(\mathrm{~m}, 1 \mathrm{H}), 2.30(\mathrm{~m}, 1 \mathrm{H}), 3.72(\mathrm{~d}, J=3.0 \mathrm{~Hz}, 1 \mathrm{H}), 3.75(\mathrm{~m}, 1 \mathrm{H}), 7.48(\mathrm{~d}, J=8.4 \mathrm{~Hz}, 2 \mathrm{H})$, $7.66(\mathrm{~d}, J=8.4 \mathrm{~Hz}, 2 \mathrm{H}) ;{ }^{13} \mathrm{C}$ NMR $\delta 12.7,25.6,28.18,28.21,46.9,51.0,105.2,128.6,130.5,133.3$, 137.8, 164.4, 165.3; FTIR (thin film): 2970 (s), 1748 (s), 1596 (w), 1494 (s), 1394 (m), 1384 (m), 1300 (s), 1209 (m), 1092 (m), 999 (m), 900 (w), 861 (w), 833 (m); MS (ESI neg.) calcd for $\mathrm{C}_{15} \mathrm{H}_{16} \mathrm{ClO}_{4}$ (M-H) 295.1 found 295.1; Anal. Calcd for $\mathrm{C}_{15} \mathrm{H}_{17} \mathrm{ClO}_{4}$ : C, 60.71; H, 5.77. Found: C, 60.64; H, 5.84. The enantioselectivity was $44 \%$ ee (Daicel Chiracel OD-H column $(25 \mathrm{~cm})$ with hexane:2-propanol=92:8, flow $0.8 \mathrm{~mL} / \mathrm{min}$, retention time: $23.8 \mathrm{~min}$; major, $29.6 \mathrm{~min}$; minor)

\section{(+)-5-[1-(4-Bromo-phenyl)-propyl]-2,2-dimethyl-[1,3]dioxane-4,6-dione (2g).}

The compound ( $34.5 \mathrm{mg}, 0.101 \mathrm{mmol})$ was obtained from $51.7 \mathrm{mg}(0.166 \mathrm{mmol})$ of the substrate $1 \mathrm{~g}$ in $61 \%$ yield as a colorless oil: $[\alpha]^{25}{ }_{\mathrm{D}}+6.00^{\circ}\left(\mathrm{c}=0.895, \mathrm{CHCl}_{3}, 34 \%\right.$ ee); ${ }^{1} \mathrm{H}$ NMR $\delta 0.92$ (t, $J=7.4 \mathrm{~Hz}$, $3 \mathrm{H}), 1.34(\mathrm{~s}, 3 \mathrm{H}), 1.67(\mathrm{~s}, 3 \mathrm{H}), 2.03(\mathrm{~m}, 1 \mathrm{H}), 2.26(\mathrm{~m}, 1 \mathrm{H}), 3.64(\mathrm{~m}, 1 \mathrm{H}), 3.68(\mathrm{~d}, J=3.0 \mathrm{~Hz}, 1 \mathrm{H}), 7.21$ (d, $J=6.9 \mathrm{~Hz}, 2 \mathrm{H}), 7.42$ (d, $J=6.9 \mathrm{~Hz}, 2 \mathrm{H}) ;{ }^{13} \mathrm{C}$ NMR $\delta 12.7,25.5,28.2,47.0,50.9,105.2,121.5,130.8$, 131.6, 138.3, 164.4, 165.2; FTIR (thin film): 1745 (s), 1489 (w), 1383 (w), 1294 (s), 1206 (m), 1075 (w), $1010(\mathrm{~m}), 830(\mathrm{w})$; MS (ESI neg.) calcd for $\mathrm{C}_{15} \mathrm{H}_{16} \mathrm{BrO}_{4}(\mathrm{M}-\mathrm{H})^{-} 339.0$ found 338.9; Anal. Calcd for $\mathrm{C}_{15} \mathrm{H}_{17} \mathrm{BrO}_{4}$ : C, 52.80; H, 5.02. Found: C, 52.86; H, 5.14.

The enantioselectivity was $45 \%$ ee (Daicel Chiracel OD-H column $(15 \mathrm{~cm})$ with hexane:2-propanol=90:10, flow $0.8 \mathrm{~mL} / \mathrm{min}$, retention time: $9.32 \mathrm{~min}$; major, $11.0 \mathrm{~min}$; minor)

\section{(+)-2,2-Dimethyl-5-(1-p-tolyl-propyl)-[1,3]dioxane-4,6-dione (2h).}

The compound ( $36.0 \mathrm{mg}, 0.130 \mathrm{mmol})$ was obtained from $40.9 \mathrm{mg}(0.166 \mathrm{mmol})$ of the substrate $1 \mathbf{h}$ in $78 \%$ yield as a white solid: $\mathrm{mp} 61-62{ }^{\circ} \mathrm{C}[\alpha]^{25}{ }_{\mathrm{D}}+2.64{ }^{\circ}\left(\mathrm{c}=1.96, \mathrm{CHCl}_{3}, 34 \%\right.$ ee) ${ }^{1} \mathrm{H}$ NMR $\delta 0.93(\mathrm{t}$, $J=7.2 \mathrm{~Hz}, 3 \mathrm{H}), 1.15(\mathrm{~s}, 3 \mathrm{H}), 1.62(\mathrm{~s}, 3 \mathrm{H}), 2.06(\mathrm{~m}, 1 \mathrm{H}), 2.26(\mathrm{~m}, 1 \mathrm{H}), 2.30(\mathrm{~s}, 3 \mathrm{H}), 3.61(\mathrm{~m}, 1 \mathrm{H}), 3.66$ (d, $J=2.4 \mathrm{~Hz}, 1 \mathrm{H}), 7.10$ (d, $J=8.2 \mathrm{~Hz}, 2 \mathrm{H}), 7.18$ (d, $J=8.2 \mathrm{~Hz}, 2 \mathrm{H}) ;{ }^{13} \mathrm{C}$ NMR $\delta 12.7,21.1,25.8,28.1$, 28.5, 47.8, 51.1, 105.4, 128.8, 129.2, 136.2, 137.2, 164.6, 165.8; FTIR (KBr): 2964 (m), 1780 (s), 1743 (s), 1514 (w), 1455 (w), 1384 (m), 1316 (m), 1298 (s), 1208 (m), 1174 (m), 1067 (m), 1007 (m), 887 (w), 861 (w); MS (ESI neg.) calcd for $\mathrm{C}_{16} \mathrm{H}_{19} \mathrm{O}_{4}(\mathrm{M}-\mathrm{H})^{-} 275.1$ found 275.1; Anal. Calcd for $\mathrm{C}_{16} \mathrm{H}_{20} \mathrm{O}_{4}$ : C, 69.55; H, 7.29. Found: C, 69.44; H, 7.25.

The enantioselectivity was $44 \%$ ee (Daicel Chiracel AD-H column $(25 \mathrm{~cm})$ with hexane:2-propanol=90:10, flow $0.8 \mathrm{~mL} / \mathrm{min}$, retention time: $12.6 \mathrm{~min}$; minor, $16.0 \mathrm{~min}$; major)

\section{(+)-2,2-Dimethyl-5-[1-(4-trifluoromethyl-phenyl)-propyl]-[1,3]dioxane-4,6-dione (2i).}

The compound (77.6 $\mathrm{mg}, 0.235 \mathrm{mmol})$ was obtained from $98.2 \mathrm{mg}(0.332 \mathrm{mmol})$ of the substrate $1 \mathbf{i}$ with the ligand 3 (5.50 mg; $9.97 \mu \mathrm{mol}, 3 \mathrm{~mol} \%)$ and $\mathrm{Cu}\left(\mathrm{O}_{2} \mathrm{CCF}_{3}\right)_{2}(962 \mu \mathrm{g}, 3.32 \mu \mathrm{mol}, 1 \mathrm{~mol} \%)$ in 


\section{Supplementary Material}

$72 \%$ yield as a colorless oil: $[\alpha]^{25}{ }_{\mathrm{D}}+3.02\left(\mathrm{c}=1.41, \mathrm{EtOH}, 62 \%\right.$ ee). ${ }^{1} \mathrm{H}$ NMR $\delta 0.93(\mathrm{t}, J=7.2 \mathrm{~Hz}, 3 \mathrm{H})$, $1.37(\mathrm{~s}, 3 \mathrm{H}), 1.67(\mathrm{~s}, 3 \mathrm{H}), 2.06(\mathrm{~m}, 1 \mathrm{H}), 2.30(\mathrm{~m}, 1 \mathrm{H}), 3.72(\mathrm{~d}, J=3.0 \mathrm{~Hz}, 1 \mathrm{H}), 3.74(\mathrm{~m}, 1 \mathrm{H}), 7.48(\mathrm{~d}$, $J=8.4 \mathrm{~Hz}, 2 \mathrm{H}), 7.56(\mathrm{~d}, J=8.4 \mathrm{~Hz}, 2 \mathrm{H}) ;{ }^{13} \mathrm{C}$ NMR $\delta 12.7,25.3,28.0,28.1,47.0,50.9,105.2,125.4$, 125.5, 129.6, 129.7, 143.5, 164.3, 164.9; FTIR (thin film): 1747 (s), 1327 (s), 1294 (m), 1166 (w), $1125(\mathrm{~m}), 1069$ (w); MS (ESI neg.) calcd for $\mathrm{C}_{16} \mathrm{H}_{16} \mathrm{~F}_{3} \mathrm{O}_{4}(\mathrm{M}-\mathrm{H})^{-} 329.1$ found 329.0; Anal. Calcd for $\mathrm{C}_{16} \mathrm{H}_{17} \mathrm{~F}_{3} \mathrm{O}_{45}$ : C, 58.18; H, 5.19. Found: C, 58.28; H, 5.20.

The enantioselectivity was $62 \%$ ee (Daicel Chiracel OD-H column $(15 \mathrm{~cm}$ ) with hexane:2-propanol=92:8, flow $1.0 \mathrm{~mL} / \mathrm{min}$, retention time: $9.31 \mathrm{~min}$ : major, $11.0 \mathrm{~min}$ : minor)

\section{(+)-2,2-Dimethyl-5-(1-naphthalen-2-yl-propyl)-[1,3]dioxane-4,6-dione (2j).}

The compound ( $43.3 \mathrm{mg}, 0.139 \mathrm{mmol})$ was obtained from $46.9 \mathrm{mg}(0.166 \mathrm{mmol})$ of the substrate $1 \mathbf{j}$ in $83 \%$ yield as a colorless oil: $[\alpha]^{25}{ }_{\mathrm{D}}+10.2^{\circ}\left(\mathrm{c}=1.615, \mathrm{CHCl}_{3}, 37 \%\right.$ ee); ${ }^{1} \mathrm{H}$ NMR $\delta 0.96(\mathrm{t}, J=7.4 \mathrm{~Hz}$, $3 \mathrm{H}), 1.10(\mathrm{~s}, 3 \mathrm{H}), 1.62(\mathrm{~s}, 3 \mathrm{H}), 2.15(\mathrm{~m}, 1 \mathrm{H}), 2.42(\mathrm{~m}, 1 \mathrm{H}), 3.78(\mathrm{~d}, J=3.3 \mathrm{~Hz}, 1 \mathrm{H}), 3.84(\mathrm{~m}, 1 \mathrm{H})$, 7.42-7.48 (m, 3H), 7.76-7.83 (m, 4H); ${ }^{13} \mathrm{C}$ NMR $\delta 12.8,25.8,28.1,28.3,48.0,51.2,105.3,125.9$, 126.0, 126.6, 127.4, 127.9, 128.2, 132.6, 133.2, 136.8, 164.7, 165.6; FTIR (thin film): 1747 (s), 1394 (w), 1383 (w), 1295 (s), 1209 (m), 997 (w); MS (ESI neg.) calcd for $\mathrm{C}_{19} \mathrm{H}_{19} \mathrm{O}_{4}(\mathrm{M}-\mathrm{H})^{-} 311.1$ found 311.0; Anal. Calcd for $\mathrm{C}_{19} \mathrm{H}_{20} \mathrm{O}_{4}$ : C, 73.06; H, 6.45. Found: C, 73.02; H, 6.71.

The enantioselectivity was $67 \%$ ee (Daicel Chiracel AD-H column $(25 \mathrm{~cm}$ ) with hexane:2-propanol=90:10, flow $0.8 \mathrm{~mL} / \mathrm{min}$, retention time: $17.2 \mathrm{~min}$; minor, $22.3 \mathrm{~min}$; major)

(+)-5-(1-Furan-2-yl-propyl)-2,2-dimethyl-[1,3]dioxane-4,6-dione (2k).

The compound $(22.4 \mathrm{mg}, 88.7 \mu \mathrm{mol})$ was obtained from $30.9 \mathrm{mg}(0.139 \mathrm{mmol})$ of the substrate $1 \mathbf{k}$ with $4.60 \mathrm{mg}(8.34 \mu \mathrm{mol})$ of ligand 3 and $804 \mu \mathrm{g}(2.78 \mu \mathrm{mol})$ of $\mathrm{Cu}\left(\mathrm{O}_{2} \mathrm{CCF}_{3}\right)_{2}$ in $64 \%$ yield as a colorless oil: $[\alpha]^{25}{ }_{\mathrm{D}}+8.71^{\circ}\left(\mathrm{c}=0.630, \mathrm{CHCl}_{3}, 76 \%\right.$ ee $) ;{ }^{1} \mathrm{H}$ NMR $\delta 1.01(\mathrm{t}, J=7.5 \mathrm{~Hz}, 3 \mathrm{H}), 1.54(\mathrm{~s}, 3 \mathrm{H})$, $1.73(\mathrm{~s}, 3 \mathrm{H}), 1.93(\mathrm{~m}, 1 \mathrm{H}), 2.21(\mathrm{~m}, 1 \mathrm{H}), 3.75(\mathrm{~d}, J=3.0 \mathrm{~Hz}, 1 \mathrm{H}), 3.79(\mathrm{~m}, 1 \mathrm{H}), 6.20(\mathrm{~d}, J=3.0 \mathrm{~Hz}, 1 \mathrm{H})$, $6.30(\mathrm{dd}, J=1.8,3.0 \mathrm{~Hz}, 1 \mathrm{H}), 7.31(\mathrm{~d}, J=1.8 \mathrm{~Hz}, 1 \mathrm{H}) ;{ }^{13} \mathrm{C}$ NMR $\delta 12.6,24.4,27.9,28.2,40.8,48.7$, 105.1, 107.3, 110.4, 141.3, 153.6, 164.2, 164.9; FTIR (thin film): 1783 (m), 1748 (s), 1384 (w), 1295 (s), 1206 (m), 1009 (w); MS (ESI neg.) calcd for $\mathrm{C}_{13} \mathrm{H}_{15} \mathrm{O}_{5}(\mathrm{M}-\mathrm{H})^{-} 251.1$ found 251.1; Anal. Calcd for $\mathrm{C}_{13} \mathrm{H}_{16} \mathrm{O}_{5}$ : C, 61.90; H, 6.39. Found: C, 61.89; H, 6.59.

The enantioselectivity was $80 \%$ ee (Daicel Chiracel AD-H column $(25 \mathrm{~cm}$ ) with hexane:2-propanol=90:10, flow $0.8 \mathrm{~mL} / \mathrm{min}$, retention time: $13.7 \mathrm{~min}$; minor, $16.1 \mathrm{~min}$; major)

(+)-2,2-Dimethyl-5-(1-thiophen-2-yl-propyl)-[1,3]dioxane-4,6-dione (2I).

The literature reported compound $\mathbf{2} \mathbf{1}^{12)}$ (32.9 $\mathrm{mg}, 0.144 \mathrm{mmol}$ ) was obtained in $87 \%$ yield from 39.6 $\mathrm{mg}(0.166 \mathrm{mmol})$ of the substrate $1 \mathrm{lin} 89 \%$ yield as a white solid: $[\alpha]^{25}+4.02^{\circ}\left(\mathrm{c}=0.905, \mathrm{CHCl}_{3}\right)$; ${ }^{1} \mathrm{H}$ NMR $\delta 0.98(\mathrm{t}, J=7.4 \mathrm{~Hz}, 3 \mathrm{H}), 1.36$ (s, 3H), 1.69 (s, 3H), $2.06(\mathrm{~m}, 1 \mathrm{H}), 2.28(\mathrm{~m}, 1 \mathrm{H}), 3.72$ (d, $J=3.0$ 


\section{Supplementary Material}

$\mathrm{Hz}, 1 \mathrm{H}), 3.98(\mathrm{~m}, 1 \mathrm{H}), 6.93(\mathrm{dd}, J=3.6,5.1 \mathrm{~Hz}, 1 \mathrm{H}), 6$. (ddd, $J=0.6,1.2,3.6 \mathrm{~Hz}, 1 \mathrm{H}), 7.18(\mathrm{dd}, J=1.2$, $5.1 \mathrm{~Hz}, 1 \mathrm{H}) ;{ }^{13} \mathrm{C}$ NMR $\delta 12.8,27.8,28.0,28.3,42.8,51.2,105.3,124.7,126.6,126.8,142.2,164.2$, 165.2; FTIR (KBr): 1779 (s), 1737 (s), 1461 (w), 1386 (w), 1308 (s), 1202 (m), 1053 (m), 1002 (m), 882 (w), 865 (w); MS (ESI neg.) calcd for $\mathrm{C}_{13} \mathrm{H}_{15} \mathrm{O}_{4} \mathrm{~S}(\mathrm{M}-\mathrm{H})^{-} 267.1$ found 267.0

The enantioselectivity was $82 \%$ ee (Daicel Chiracel OD-H column $(15 \mathrm{~cm})$ with hexane:2-propanol=92:8, flow $1 \mathrm{~mL} / \mathrm{min}$, retention time: $12.5 \mathrm{~min}$ : minor, $15.2 \mathrm{~min}$ : major).

References:

1) Alexakis, A.; Rosset, S.; Allamand, J.; March, S; Guillen, F.; Benhaim, C. Synlett 2001, $1375-1378$.

2) Iseki, K.; Mizuno, S.; Kuroki, Y.; Kobayashi, Y. Tetrahedron, 1999, 55, 977-988.

3) Bigi, F.; Carloni, S.; Ferrari, L.; Maggi, R.; Mazzacani, A.; Sartoni, G. Tetrahedron Lett., 2001, 42, 5203-5205.

4) Schuster, P.; Polansky, O. E.; Wessely, F. Monatsh. Chem., 1964, 95, 53-58.

5) Shi, D.; Wang, Y.; Lu, Z.; Dai, G. Synth. Commun,. 2000, 30, 713-726.

6) Cornélis, A.; Lambert, S.; Laszlo, P.; Schaus, P. J. Org. Chem., 1981, 46, 2130-2134.

7) Kunz, F. J.; Margaretha, P.; Polansky, O. E. Chimia, 1970, 24, 165-181.

8) Mass, S.; Stamm, A.; Kunz, H. Synthesis, 1999, 1792-1798.

9) Enders, D.; Rendenbach, B. E. M. Tetrahedron, 1986, 42, 2235-2242.

10) Myers, A. I.; Smith, R. K.; Whitten, C. E. J. Org. Chem., 1979, 44, 2250-2256.

11) Shintani, R.; Fu, G. C. Org. Lett., 2002, 4, 3699-3702.

12) Haslego, M. L.; Smith, F. X. Synth. Commun., 1980, 10, 421-427. 